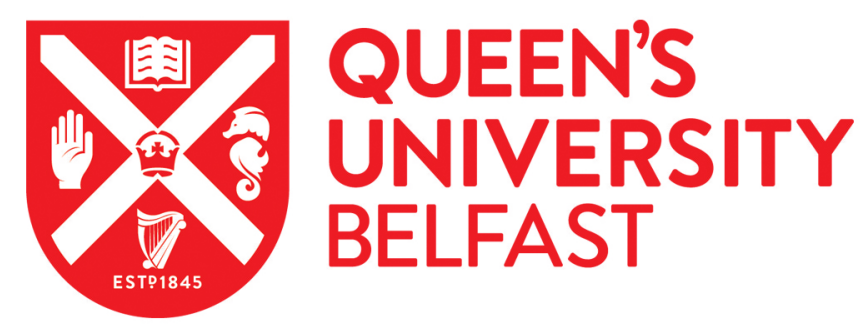

\title{
Optimal Power Allocation for Multiuser Secure Communication in Cooperative Relaying Networks
}

Duong, T. Q., Hoang, T. M., Kundu, C., Elkashlan, M., \& Nallanathan, A. (2016). Optimal Power Allocation for Multiuser Secure Communication in Cooperative Relaying Networks. IEEE Wireless Communications Letters, 5(5), 516-519. https://doi.org/10.1109/LWC.2016.2597278

\author{
Published in: \\ IEEE Wireless Communications Letters
}

\section{Document Version:}

Peer reviewed version

\section{Queen's University Belfast - Research Portal:}

Link to publication record in Queen's University Belfast Research Portal

\begin{abstract}
Publisher rights
Copyright 2016 IEEE. Personal use of this material is permitted. Permission from IEEE must be obtained for all other users, including reprinting/ republishing this material for advertising or promotional purposes, creating new collective works for resale or redistribution to servers or lists, or reuse of any copyrighted components of this work in other works
\end{abstract}

\section{General rights}

Copyright for the publications made accessible via the Queen's University Belfast Research Portal is retained by the author(s) and / or other copyright owners and it is a condition of accessing these publications that users recognise and abide by the legal requirements associated with these rights.

\section{Take down policy}

The Research Portal is Queen's institutional repository that provides access to Queen's research output. Every effort has been made to ensure that content in the Research Portal does not infringe any person's rights, or applicable UK laws. If you discover content in the Research Portal that you believe breaches copyright or violates any law, please contact openaccess@qub.ac.uk. 


\title{
Optimal Power Allocation for Multiuser Secure Communication in Cooperative Relaying Networks
}

\author{
Trung Q. Duong, Tiep M. Hoang, Chinmoy Kundu, Maged Elkashlan, and Arumugam Nallanathan
}

\begin{abstract}
We consider a cooperative relaying network in which a source communicates with a group of users in the presence of one eavesdropper. We assume that there are no source-user links and the group of users receive only retransmitted signal from the relay. Whereas, the eavesdropper receives both the original and retransmitted signals. Under these assumptions, we exploit the user selection technique to enhance the secure performance. We first find the optimal power allocation strategy when the source has the full channel state information (CSI) of all links. We then evaluate the security level through: i) ergodic secrecy rate and ii) secrecy outage probability when having only the statistical knowledge of CSIs.
\end{abstract}

Index Terms-Physical layer security, secrecy capacity, decodeand-forward, best user selection.

\section{INTRODUCTION}

Physical layer security has emerged and attracted the attention of communication society widely over the past decades. To deal with the leakage effect, many efforts to guarantee the security of cooperative networks have been made so far. Particularly, selection techniques are studied for the purpose of selecting suitable nodes in order to perform effective and safe transmission. Such selection techniques can be relay selection [1], jamming selection [2], [3], antenna selection [4], and user selection [5]. In general, such diverse selection techniques have been widely studied in literature.

Among the aforementioned techniques, user selection is an appropriate scheme for multiuser communications [5]. It exploits cooperative users to capture the cooperative diversity from a user group and enhance system security. We thus employ the user selection technique for choosing the best link among all links from a single relay to multiple users. Compared to closely previous related works, the differences are major, for example [5] exploited the user selection technique but did not discuss the source-eavesdropper link; while [6] did not discuss the impact of multiple users although the model is similar to ours. In contrast, we consider a severe scenario with the existence of source-eavesdropper link but no source-destination link. As such, the destination is in a much weaker position than the eavesdropper because the destination is receiving only one signal from the relay. We are thus

T. Q. Duong and C. Kundu are with Queen's University Belfast, UK (e-mail: \{trung.q.duong, c.kundu@qub.ac.uk\}@qub.ac.uk). T. M. Hoang is with Duy Tan University, Vietnam (e-mail: hmt1803@gmail.com). M. Elkashlan is with Queen Mary University of London, UK (e-mail: maged.elkashlan@qmul.ac.uk). A. Nallanathan is with King's College London, UK (e-mail: arumugam.nallanathan@kcl.ac.uk).

This work was supported by the U.K. Royal Academy of Engineering Research Fellowship under Grant RF1415 $\backslash 14 \backslash 22$, by the Newton Institutional Link under Grant ID 172719890, and by the Royal Society-SERB Newton International Fellowship under Grant ID NF151345. motivated to examine such a secure cooperative network to understand the impact of the direct eavesdropping link on the secure performance. First, with the knowledge of instantaneous channel state information (CSI) of every channel, we find the optimal instantaneous source power which maximizes the instantaneous secrecy rate (SR). Then, only with the statistical knowledge of CSI, we evaluate the security level through the ergodic SR and secrecy outage probability (SOP).

\section{System Model And User Selection CRiterion}

\section{A. System Model}

We consider the multiuser secure communication in cooperative relay networks, in which there are one source, one relay, one eavesdropper and multiple destinations, and all the nodes are single-antenna devices operating in the half-duplex mode. For notational simplicity, we denote the source, the relay, the $k$-th destination and the eavesdropper by $\mathrm{S}, \mathrm{R}, k \mathrm{D}$ (or simply $k$ ) and $\mathrm{E}$ respectively. We assume that there is no direct link between $\mathrm{S}$ and $k \mathrm{D}$ due to loss and shadowing. In the first time slot, $\mathrm{S}$ broadcasts its signal while $\mathrm{R}$ attempts to decode the source signal. In the second time slot, $\mathrm{R}$ forwards the decoded signal to the best destination. We note that the signals transmitted by $\mathrm{S}$ and $\mathrm{R}$ are intercepted by $\mathrm{E}$. Moreover, we assume that the CSI of $k \mathrm{D}$ as well as that of $\mathrm{E}$ is available at $\mathrm{S}$ and R. Herein, the availability of the CSI of E is a commonly used assumption when $\mathrm{E}$ is an active user of the system and not the intended destination for confidential messages [3], [4].

The channel between $X \in\{S, R\}$ and $Y \in\{R, k D, E\}$ (with $Y \neq X$ ) is assumed to suffer from block Rayleigh fading with the channel gain $h_{X Y} \sim \mathcal{C N}\left(0, \Omega_{X Y}\right)^{1}$. Let $\gamma_{X Y}$ denote the instantaneous signal-to-noise ratio (SNR) at $\mathrm{Y}$ for the signal transmitted by $\mathrm{X}$. Then, we have $\gamma_{X Y}=$ $\frac{P_{X}}{N_{Y}}\left|h_{X Y}\right|^{2} \sim \operatorname{Exp}\left(\frac{P_{X}}{N_{Y}} \Omega_{X Y}\right)^{2}$ with $P_{X}$ being the transmit power at $X$ and $N_{Y}$ being the additive white Gaussian noise at $Y$. By normalizing $N_{Y}$ (i.e., $N_{Y}=0 \mathrm{~dB}$ ), $P_{X}$ can be understood as a replacement for $\frac{P_{X}}{N_{Y}}$. As such, in the first time slot, we have $\gamma_{S R} \sim \operatorname{Exp}\left(\bar{\gamma}_{S R}\right)$ and $\gamma_{S E} \sim \operatorname{Exp}\left(\bar{\gamma}_{S E}\right)$ where $\bar{\gamma}_{S R}=P_{S} \Omega_{S R}$ and $\bar{\gamma}_{S E}=P_{S} \Omega_{S E}$. Similarly, in the second time slot, we have $\gamma_{R k} \sim \operatorname{Exp}\left(\bar{\gamma}_{R D}\right)$ and $\gamma_{R E} \sim \operatorname{Exp}\left(\bar{\gamma}_{R E}\right)$ where $\bar{\gamma}_{R D}=P_{R} \Omega_{R D}$ and $\bar{\gamma}_{R E}=P_{R} \Omega_{R E}$.

\section{B. User Selection Criterion}

In this paper, the best user link selection is proposed, in which the destination corresponding to $\max _{k}\left\{\gamma_{R k}\right\}$ is selected

\footnotetext{
${ }^{1} \mathcal{C N}(0, \Omega)$ denotes a complex Gaussian variable with zero-mean and variance $\Omega$.

${ }^{2} \operatorname{Exp}(m)$ denotes the exponential distribution with mean $m$.
} 
for receiving the signal retransmitted by $\mathrm{R}$. We use $k^{*} \mathrm{D}$ to denote the selected/strongest $\mathrm{D}$ corresponding to the index $k^{*}=\arg \max _{k} \gamma_{R k}$. The instantaneous SNR for the R-k*D channel is therefore $\gamma_{R k^{*}}=\max _{k} \gamma_{R k}$.

Proposition 1. The CDF and PDF of $\gamma_{R k^{*}}$ can be, respectively, given by

$$
\begin{aligned}
& F_{\gamma_{R k^{*}}}(\gamma)=\left(1-e^{-\gamma / \bar{\gamma}_{R D}}\right)^{K}=1-\widetilde{\sum_{k}} e^{-\left(k / \bar{\gamma}_{R D}\right) \gamma} \\
& f_{\gamma_{R k^{*}}}(\gamma)=d F_{\gamma_{R k^{*}}}(\gamma) / d \gamma=\widetilde{\sum_{k}}\left(k / \bar{\gamma}_{R D}\right) e^{-\left(k / \bar{\gamma}_{R D}\right) \gamma} .
\end{aligned}
$$

where $\widetilde{\sum_{k}}(\cdot) \triangleq \sum_{k=1}^{K}\left(\begin{array}{c}K \\ k\end{array}\right)(-1)^{k-1}(\cdot)$.

\section{Instantaneous Source Power Allocation STRATEGY}

In this section, we examine the instantaneous source power which maximizes the SR. It should also be noted that the SR is the difference between the capacity of the desired link and that of eavesdropping link. Thus, for realistic scenarios, we only consider the worst-case scenario where $\mathrm{E}$ is able to maximize the probability of successful eavesdropping.

The selected $k^{*} \mathrm{D}$ only receives the signal retransmitted by $\mathrm{R}$, the end-to-end capacity of the channel from $\mathrm{S}$ to $k^{*} \mathrm{D}$ with the help of $\mathrm{R}$ is given by

$$
C_{k^{*}}=(1 / 2) \log _{2}\left(1+\min \left\{\gamma_{S R}, \gamma_{R k^{*}}\right\}\right) .
$$

While $\mathrm{E}$ receives both versions of $x$, the end-to-end capacity of the channel from $\mathrm{S}$ to $\mathrm{E}$ with the help of $\mathrm{R}$ is given by ${ }^{3}$

$$
C_{E}=(1 / 2) \log _{2}\left(1+\min \left\{\gamma_{S R}, \gamma_{S E}+\gamma_{R E}\right\}\right) .
$$

For the sake of convenience, we let $X \triangleq \min \left\{\gamma_{S R}, \gamma_{R k^{*}}\right\}$ and $Y \triangleq \min \left\{\gamma_{S R}, \gamma_{S E}+\gamma_{R E}\right\}$.

We assume that CSIs of all channels are perfectly known (full CSIs). The instantaneous SR of the system can be defined as [6]

$$
C_{\Delta}=\left[C_{k^{*}}-C_{E}\right]^{+}=(1 / 2)\left[\log _{2}((1+X) /(1+Y))\right]^{+}
$$

where $[x]^{+}=\max \{0, x\}$. We now consider finding the optimal transmit power $P_{S}$ that maximizes the SR given the maximum transmit power $P_{\max }$ at S. Thus our SR maximization problem can be formulated as follows:

$$
\text { (P1) } \begin{array}{ll}
\underset{0<P_{S} \leq P_{\max }}{\operatorname{maximize}} & \mathcal{U}\left(P_{S}\right) \triangleq(1+X) /(1+Y) \\
\text { subject to } & 0<P_{R} \leq P_{\max } \\
& X=\min \left\{\gamma_{S R}, \gamma_{R k^{*}}\right\} \\
& Y=\min \left\{\gamma_{S R}, \gamma_{S E}+\gamma_{R E}\right\}
\end{array}
$$

\footnotetext{
${ }^{3}$ The end-to-end capacity of the channel from $\mathrm{S}$ to $\mathrm{E}$ with the participation of $\mathrm{R}$ originates from the formulation of the mutual information associated with Protocol II in [7]. A summary of this formulation is as follows: Let $r_{1}$ and $r_{2}$ be the transmission rates over the first and second time slots, respectively. Firstly, for successful decoding at R, the constraint (C1), i.e., $r_{1} \leq \log _{2}\left(1+\gamma_{S R}\right) \triangleq r_{\text {relay }}^{\max }$, needs to be satisfied. Secondly, the three nodes $\{\mathrm{S}, \mathrm{R}, \mathrm{E}\}$ form a multiple-access channel (MAC) [8], [9] in which $\mathrm{E}$ is the common receiver/eavesdropper, thus there are three constraints on $r_{1}, r_{2}$, and $r_{1}+r_{2}$ for successful decoding at $\mathrm{E}$. These three constraints, however, can be further shortened to the constraint (C2), i.e., $r_{1} \leq \log _{2}\left(1+\gamma_{S E}+\gamma_{R E}\right) \triangleq r_{1}^{\max }$, because $\mathrm{S}$ is silent during the second time slot $\left(r_{2}=0\right)$. Finally, combining $(\mathbf{C 1})$ with $(\mathbf{C} 2)$ and using the fact that transmission occurs over two time slots, (4) can be easily deduced from [7].
}

Let us label event $\mathcal{X}$ as $X=\gamma_{S R} \Leftrightarrow\left|h_{S R}\right|^{2} P_{S} \leq$ $\left|h_{R k^{*}}\right|^{2} P_{R}$, event $\mathcal{Y}$ as $Y=\gamma_{S R} \Leftrightarrow\left|h_{R E}\right|^{2} P_{R} \geq$ $\left(\left|h_{S R}\right|^{2}-\left|h_{S E}\right|^{2}\right) P_{S}$, and event $\mathcal{Z}$ as $\left|h_{S R}\right|^{2} \leq\left|h_{S E}\right|^{2}$ respectively. In contrast, $\overline{\mathcal{X}}, \overline{\mathcal{Y}}$, and $\overline{\mathcal{Z}}$ denote events $X=\gamma_{R k^{*}}$, $Y=\gamma_{S E}+\gamma_{R E}$, and $\left|h_{S R}\right|^{2}>\left|h_{S E}\right|^{2}$ respectively.

Likewise, we label event $\mathcal{M}$ as $P_{\max } \geq \frac{\left|h_{R k^{*}}\right|^{2} P_{R}}{\left|h_{S R}\right|^{2}} \triangleq \mathcal{P}_{\mathcal{M}}$ and event $\mathcal{N}$ as $P_{\max } \geq \frac{\left|h_{R E}\right|^{2} P_{R}}{\left|h_{S R}\right|^{2}-\left|h_{S E}\right|^{2}} \triangleq \mathcal{P}_{\mathcal{N}}$ respectively. While $\overline{\mathcal{M}}, \overline{\mathcal{N}}$ denote events $P_{\max }<\mathcal{P}_{\mathcal{M}}$ and $P_{\max }<\mathcal{P}_{\mathcal{N}}$ respectively.

Once events are coupled together, new joint events arise. For the sake of convenience, we provide Tables I and II, which present the new joint conditions below.

TABLE I

JoInt EVEnts of $\{\mathcal{X}, \overline{\mathcal{X}}\}$, AND $\{\mathcal{M}, \overline{\mathcal{M}}\}$

\begin{tabular}{|c|c|c|}
\hline & $\mathcal{M}$ & $\mathcal{M}$ \\
\hline $\mathcal{X}$ & $0 \leq P_{S} \leq \mathcal{P}_{\mathcal{M}} \leq P_{\max }$ & $0 \leq P_{S} \leq P_{\max }$ \\
\hline $\mathcal{X}$ & $0<\mathcal{P}_{\mathcal{M}}<P_{S} \leq P_{\max }$ & null \\
\hline
\end{tabular}

TABLE II

JoInt EVENTS OF $\{\mathcal{Y}, \overline{\mathcal{Y}}\},\{\mathcal{Z}, \overline{\mathcal{Z}}\}$, AND $\{\mathcal{N}, \overline{\mathcal{N}}\}$

\begin{tabular}{|c|c|c|c|}
\hline & $\mathcal{Z}$ & $\overline{\mathcal{Z}}$ and $\mathcal{N}$ & $\overline{\mathcal{Z}}$ and $\overline{\mathcal{N}}$ \\
\hline $\mathcal{Y}$ & $0 \leq P_{S} \leq P_{\max }$ & $0 \leq P_{S} \leq \mathcal{P}_{\mathcal{N}} \leq P_{\max }$ & $0 \leq P_{S} \leq P_{\max }$ \\
\hline$\overline{\mathcal{Y}}$ & null & $0<\mathcal{P}_{\mathcal{N}}<P_{S} \leq P_{\max }$ & null \\
\hline
\end{tabular}

1) Scenario $1(\mathcal{X} \cap \mathcal{Y})$ : The objective function becomes $\mathcal{U}\left(P_{S}\right)=\frac{1+\gamma_{S R}}{1+\gamma_{S R}}=1$, which leads to $C_{\Delta}=0$ regardless of $P_{S}$ and $P_{R}$. It reveals that if any transmission is performed, it is not effective. Thus, $\mathrm{S}$ should not transmit signal, i.e., $P_{S}^{(1)}=0$.

2) Scenario $2(\mathcal{X} \cap \overline{\mathcal{Y}})$ : The objective function becomes $\mathcal{U}\left(P_{S}\right)=\frac{1+\gamma_{S R}}{1+\gamma_{S E}+\gamma_{R E}}$. The first order derivative of $\mathcal{U}\left(P_{S}\right)$ is then shown as $\frac{\partial \mathcal{U}\left(P_{S}\right)}{\partial P_{S}}=\frac{P_{R}\left|h_{R E}\right|^{2}\left|h_{S R}\right|^{2}+\left|h_{S R}\right|^{2}-\left|h_{S E}\right|^{2}}{\left(1+\gamma_{S E}+\gamma_{R E}\right)^{2}}$. Thus, $\mathcal{U}\left(P_{S}\right)$ is a monotonically non-decreasing function when

$$
\left(\left|h_{S E}\right|^{2}-\left|h_{S R}\right|^{2}\right) /\left(\left|h_{R E}\right|^{2}\left|h_{S R}\right|^{2}\right) \leq P_{R} .
$$

Moreover, from Tables I and II we can see that the joint event $\mathcal{X} \cap \overline{\mathcal{Y}}$ occurs if and only if the event $\mathcal{X}, \overline{\mathcal{Z}}$, and $\mathcal{N}$ occur simultaneously. Due to $\overline{\mathcal{Z}} \Leftrightarrow\left|h_{S R}\right|^{2}>\left|h_{S E}\right|^{2}$ and $0 \leq P_{R}$, the condition (6) is always true. In this case, $\mathcal{U}\left(P_{S}\right)$ is always monotonically non-decreasing, thus $P_{S}$ should be allocated its largest possible value. Looking back to Tables I and II, we deduce that the optimal value of $P_{S}$, i.e. $P_{S}^{o p t}$, is divided into two cases: i) If the event $\mathcal{M} \cap \overline{\mathcal{Y}}$ occurs, then $P_{S}^{o p t}=\mathcal{P}_{\mathcal{M}}$; ii) If the event $\overline{\mathcal{M}} \cap \overline{\mathcal{Y}}$ occurs, then $P_{S}^{\text {opt }}=P_{\max }$. In other words, the optimal transmit power in this scenario is given by $P_{S}^{(2)}=\min \left\{\mathcal{P}_{\mathcal{M}}, P_{\max }\right\}$.

3) Scenario $3(\overline{\mathcal{X}} \cap \mathcal{Y})$ : The objective function becomes $\mathcal{U}\left(P_{S}\right)=\frac{1+\gamma_{R k^{*}}}{1+\gamma_{S R}}$, which decreases inversely with $P_{S}$. Thus, $\mathcal{U}\left(P_{S}\right)$ gets maximum when $P_{S}$ reaches its minimum. Using Tables I and II, we can readily find that the optimal transmit power in the scenario $\overline{\mathcal{X}} \cap \mathcal{Y}$ is given by $P_{S}^{(3)}=\max \left\{0, \mathcal{P}_{\mathcal{M}}\right\}$.

4) Scenario $4(\overline{\mathcal{X}} \cap \overline{\mathcal{Y}})$ : The objective function becomes $\mathcal{U}\left(P_{S}\right)=\frac{1+\gamma_{R k^{*}}}{1+\gamma_{S E}+\gamma_{R E}}$, which decreases inversely with $P_{S}$. Similar to the scenario $\overline{\mathcal{X}} \cap \mathcal{Y}$, we can find the optimal transmit power by using Tables I and II, that is $P_{S}^{(4)}=\max \left\{\mathcal{P}_{\mathcal{M}}, \mathcal{P}_{\mathcal{N}}\right\}$. 
Finally, the constraint $0<P_{S} \leq P_{\max }$ of $(\mathbf{P 1})$ is equivalent to $P_{S} \in\left\{P_{S}^{(i)} \mid i=1,2,3,4\right\}$, i.e.,

$$
\begin{aligned}
& \text { (P1) } \operatorname{maximize}_{0<P_{S} \leq P_{\max }} \mathcal{U}\left(P_{S}\right) \\
& \Leftrightarrow(\mathbf{P 2}) \underset{P_{S} \in\left\{P_{S}^{(i)} \mid i=1,2,3,4\right.}{\operatorname{maximize}} \mathcal{U}\left(P_{S}\right) .
\end{aligned}
$$

As such, $(\mathbf{P} 2)$ can be readily solved by choosing the optimal $P_{S}^{(i)}, i \in\{1,2,3,4\}$, so that $U\left(P_{S}^{(i)}\right)$ is maximal.

\section{Performance Analysis}

In this section, we evaluate the secure performance of our system model through two metrics: i) ergodic SR and ii) SOP. These two metrics are derived without requiring the knowledge of the instantaneous CSIs.

\section{A. Ergodic Secrecy Rate}

The ergodic SR in bits/s/Hz is given by

$$
\begin{aligned}
\langle C\rangle & =(2 \ln 2)^{-1} \mathbb{E}_{\gamma_{S R}}\left\{\mathbb{E}_{Y \mid \gamma_{S R}}\left\{\omega_{1}(y, \gamma) \mid \gamma_{S R}=\gamma\right\}\right\} \\
& =(2 \ln 2)^{-1} \mathbb{E}_{\gamma_{S R}}\left\{\omega_{2}(\gamma)\right\}
\end{aligned}
$$

where

$$
\begin{aligned}
\omega_{1}(y, \gamma) & \triangleq \int_{0}^{\infty}\left[\ln \left(\frac{1+x}{1+y}\right)\right]^{+} f_{X \mid \gamma_{S R}}(x \mid \gamma) d x, \\
\omega_{2}(\gamma) & \triangleq \int_{0}^{\infty} \omega_{1}(y, \gamma) f_{Y \mid \gamma_{S R}}(y \mid \gamma) d y .
\end{aligned}
$$

The second equality of (7) follows from that $X \mid \gamma_{S R}$ and $Y \mid \gamma_{S R}$ are independent. Their distributions are respectively shown in Propositions 2 and 3.

Proposition 2. The CDF of $X \mid \gamma_{S R}=$ $\min \left\{\gamma_{S R}, \gamma_{R k^{*}} \mid \gamma_{S R}=\gamma\right\}$ is given by

$$
F_{X \mid \gamma_{S R}}(x)=\left\{\begin{array}{ll}
F_{\gamma_{R k^{*}}}(x), & \text { if } x<\gamma \\
1, & \text { if } x \geq \gamma
\end{array} .\right.
$$

It is noted that $X \mid \gamma_{S R}$ is a mixed random variable, thus its PDF can be calculated as [10, Chapter 3]

$$
f_{X \mid \gamma_{S R}}(x)=f_{\gamma_{R k^{*}}}(x)+\left[1-F_{\gamma_{R k^{*}}}(\gamma)\right] \delta(x-\gamma),
$$

if $x \leq \gamma$, where $\delta(x-\gamma)$ is a Dirac delta function in $x$.

Proposition 3. Let $Y_{0}=\gamma_{S E}+\gamma_{R E}$, then the CDF and PDF of $Y_{0}$ can be, respectively, given by

$$
\begin{aligned}
& F_{Y_{0}}(y)=1-\frac{\left(\bar{\gamma}_{S E} e^{-y / \bar{\gamma}_{S E}}-\bar{\gamma}_{R E} e^{-y / \bar{\gamma}_{R E}}\right)}{\bar{\gamma}_{S E}-\bar{\gamma}_{R E}}, \\
& f_{Y_{0}}(y)=\left(e^{-y / \bar{\gamma}_{S E}}-e^{-y / \bar{\gamma}_{R E}}\right)\left(\bar{\gamma}_{S E}-\bar{\gamma}_{R E}\right)^{-1} .
\end{aligned}
$$

Let $Y \mid \gamma_{S R}=\min \left\{\gamma_{S R}, Y_{0} \mid \gamma_{S R}=\gamma\right\}$, we can obtain the CDF and the PDF of $Y \mid \gamma_{S R}$ by replacing $\gamma_{R k^{*}}$ in (10)-(11) with $Y_{0}$.

Substituting (11) into (8) and using the sifting property of Dirac delta function, we have

$$
\omega_{1}(y, \gamma)=\widetilde{\sum_{k}} e^{\frac{k}{\bar{\gamma}_{R D}}}\left[E_{1}\left(\frac{k(1+y)}{\bar{\gamma}_{R D}}\right)-E_{1}\left(\frac{k(1+\gamma)}{\bar{\gamma}_{R D}}\right)\right]
$$

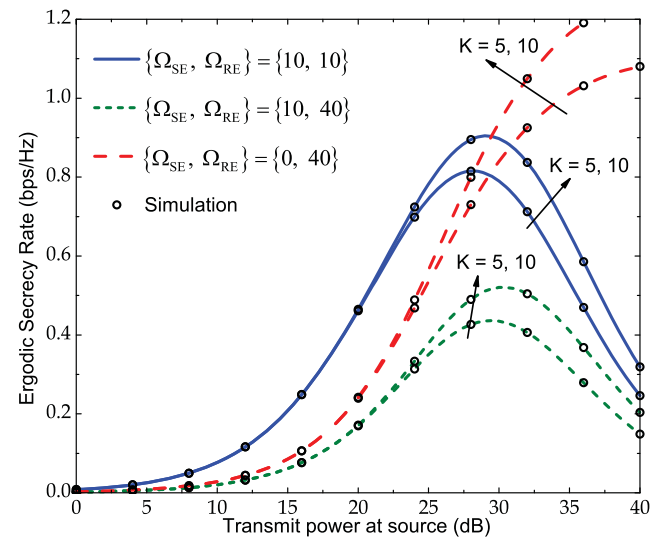

Fig. 1. $\langle C\rangle$ as a function of $\frac{P_{S}}{N_{0}}$ (or $P_{S}$ ). Other system parameters are as follows: $\frac{P_{\max }}{N_{0}}=40 \mathrm{~dB}, \frac{P_{R}}{N_{0}} \approx 26.99 \mathrm{~dB}, \Omega_{S R}=80$, and $\Omega_{R D}=50$.

where $E_{1}(x)=\int_{x}^{\infty} \frac{e^{-u}}{u} d u$ for $y \leq \gamma$; otherwise, $\omega_{1}(y, \gamma)=0$ for $y>\gamma$. As such, $\omega_{2}(\gamma)$ in (9) can be reduced to $\omega_{2}(\gamma)=$ $\int_{0}^{\gamma} \omega_{1}(y, \gamma) f_{Y_{0}}(y) d y$ by integrating over $y \leq \gamma$. Using (13) and (14) to evaluate $\omega_{2}(\gamma)$ again, we then arrive at

$$
\begin{aligned}
\omega_{2}(\gamma)= & \widetilde{\sum_{k}} e^{\frac{k}{\bar{\gamma}_{R D}}}\left(\bar{\gamma}_{S E}-\bar{\gamma}_{R E}\right)^{-1} \\
\times & \left\{\int_{0}^{\gamma} E_{1}\left(k(1+y) / \bar{\gamma}_{R D}\right)\left(e^{-\frac{y}{\bar{\gamma}_{S E}}}-e^{-\frac{y}{\bar{\gamma}_{R E}}}\right) d y\right. \\
& +E_{1}\left(k(1+\gamma) / \bar{\gamma}_{R D}\right)\left(\bar{\gamma}_{S E} e^{-\frac{\gamma}{\bar{\gamma}_{S E}}}-\bar{\gamma}_{R E} e^{-\frac{\gamma}{\bar{\gamma}_{R E}}}\right) \\
& \left.-E_{1}\left(k(1+\gamma) / \bar{\gamma}_{R D}\right)\left(\bar{\gamma}_{S E}-\bar{\gamma}_{R E}\right)\right\} .
\end{aligned}
$$

Finally, substituting (15) into (7) and using [11, Eq. (5.231.2)], we obtain the ergodic SR as shown at the bottom of the next page.

\section{B. Secrecy Outage Probability}

The SOP is given by

$$
\mathcal{P}_{\text {out }}(\zeta)=\mathbb{P}\left\{C_{\Delta} \leq R\right\}=\int_{0}^{\infty} P(\gamma, \zeta) f_{\gamma_{S R}}(\gamma) d \gamma
$$

where $\zeta=2^{2 R}-1$ and the term $P(\gamma, \zeta)$ is defined as

$$
\begin{aligned}
P(\gamma, \zeta) \triangleq & \mathbb{P}\left\{X-(\zeta+1) Y \leq \zeta \mid \gamma_{S R}=\gamma\right\} \\
= & \int_{0}^{\gamma} F_{X \mid \gamma_{S R}}((\zeta+1) y+\zeta) \\
& \quad \times\left[f_{Y_{0}}(y)+\left(1-F_{Y_{0}}(\gamma)\right) \delta(y-\gamma)\right] d y
\end{aligned}
$$

where $f_{Y \mid \gamma_{S R}}(y \mid \gamma)$, along with $f_{Y_{0}}(y)$, is presented in Proposition 3. Combining (18) with (10), we then have two cases:

- If $0 \leq \gamma \leq \zeta$, we deduce from (10) that $F_{X \mid \gamma_{S R}}((\zeta+1) y+\zeta)=1$ and hence (18) becomes

$$
P(\gamma, \zeta)=F_{Y_{0}}(\gamma)+\left(1-F_{Y_{0}}(\gamma)\right)=1 \text {. }
$$

- If $\zeta<\gamma<\infty$, we deduce from (10) that $F_{X \mid \gamma_{S R}}((\zeta+1) y+\zeta)=F_{\gamma_{R k^{*}}}((\zeta+1) y+\zeta)$ if $y<$ $\frac{\gamma-\zeta}{\zeta+1}$, and $F_{X \mid \gamma_{S R}}((\zeta+1) y+\zeta)=1$ if $y \geq \frac{\gamma-\zeta}{\zeta+1}$. Hence (18) becomes

$$
P(\gamma, \zeta)=\mathcal{J}(\gamma, \zeta)+1-F_{Y_{0}}\left(\frac{\gamma-\zeta}{\zeta+1}\right)
$$




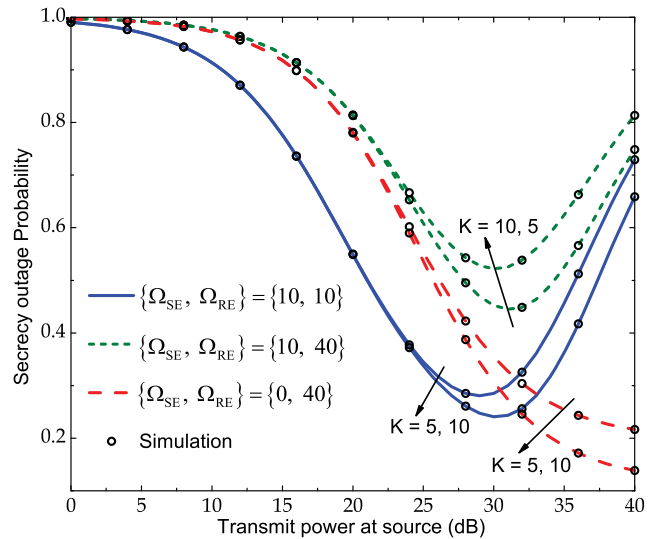

Fig. 2. $\mathcal{P}_{\text {out }}$ as a function of $\frac{P_{S}}{N_{0}}$ (or $P_{S}$ ). Other system parameters are as follows: $\frac{P_{\max }}{N_{0}}=40 \mathrm{~dB}, P_{R} / N_{0} \approx 26.99 \mathrm{~dB}, R=0.25 \mathrm{bps} / \mathrm{Hz}, \Omega_{S R}=$ 80 , and $\Omega_{R D}=50$.

where

$$
\begin{aligned}
& \mathcal{J}(\gamma, \zeta)=\left[\bar{\gamma}_{S E}-\bar{\gamma}_{R E}\right]^{-1} \widehat{\sum_{k}} e^{-\frac{k \zeta}{\bar{\gamma}_{R D}}} \\
& \times\left\{\left[1-e^{-\left(\frac{k(\gamma-\zeta)}{\bar{\gamma}_{R D}}+\frac{\gamma-\zeta}{(\zeta+1) \bar{\gamma}} S E\right.}\right)\right]\left[\frac{k(\zeta+1)}{\bar{\gamma}_{R D}}+\frac{1}{\bar{\gamma}_{S E}}\right]^{-1} \\
& \left.-\left[1-e^{-\left(\frac{k(\gamma-\zeta)}{\bar{\gamma}_{R D}}+\frac{\gamma-\zeta}{(\zeta+1) \bar{\gamma}_{R E}}\right)}\right]\left[\frac{k(\zeta+1)}{\bar{\gamma}_{R D}}+\frac{1}{\bar{\gamma}_{R E}}\right]^{-1}\right\}
\end{aligned}
$$

with $\widehat{\sum}_{k}(\cdot) \triangleq \sum_{k=0}^{K}\left(\begin{array}{c}K \\ k\end{array}\right)(-1)^{k}(\cdot)$.

Proceeding to analyze the integral (17) with the help of (19)-(20), we can rewrite (17) as

$$
\mathcal{P}_{\text {out }}(\zeta)=1-\mathcal{I}_{1}(\zeta)+\mathcal{I}_{2}(\zeta)
$$

where

$$
\begin{aligned}
\mathcal{I}_{1}(\zeta)= & \frac{\bar{\gamma}_{S R}^{2} e^{-\zeta / \bar{\gamma}_{S R}}}{\left[\bar{\gamma}_{S R}+(\zeta+1) \bar{\gamma}_{S E}\right]\left[\bar{\gamma}_{S R}+(\zeta+1) \bar{\gamma}_{R E}\right]} \\
\mathcal{I}_{2}(\zeta)= & {\left[\bar{\gamma}_{S R}\left(\bar{\gamma}_{S E}-\bar{\gamma}_{R E}\right)\right]^{-1} \sum_{k} e^{-\zeta\left(\frac{k}{\bar{\gamma}_{R D}}+\frac{1}{\bar{\gamma}_{S R}}\right)} } \\
\times & \left\{\left[\bar{\gamma}_{S R}-\left(k / \bar{\gamma}_{R D}+1 /\left((\zeta+1) \bar{\gamma}_{S E}\right)+1 / \bar{\gamma}_{S R}\right)^{-1}\right]\right. \\
& \times\left[k(\zeta+1) / \bar{\gamma}_{R D}+1 / \bar{\gamma}_{S E}\right]^{-1} \\
- & {\left[\bar{\gamma}_{S R}-\left(k / \bar{\gamma}_{R D}+1 /\left((\zeta+1) \bar{\gamma}_{R E}\right)+1 / \bar{\gamma}_{S R}\right)^{-1}\right] } \\
& \left.\times\left[k(\zeta+1) / \bar{\gamma}_{R D}+1 / \bar{\gamma}_{R E}\right]^{-1}\right\}
\end{aligned}
$$

\section{RESUlts}

In this section, we provide some numerical results to validate analytical results. Unless specifically stated, we set parameters $\frac{P_{\max }}{N_{0}}=40 \mathrm{~dB}$ and $\frac{P_{R}}{N_{0}} \approx 26.99 \mathrm{~dB}$. Recall that $\frac{P_{S}}{N_{0}}$ is considered as $P_{S}$ due to $N_{0}$ being set to $0 \mathrm{~dB}$.
Fig. 1 shows the ergodic SR $\langle C\rangle$ while Fig. 2 shows the SOP $\mathcal{P}_{\text {out }}$. In both figures, we use the same parameters $\Omega_{S R}=80$, $\Omega_{R D}=50, \Omega_{R E}=20, \Omega_{S E}=\{0,10\}, \Omega_{R E}=\{10,40\}$, and $K=\{5,10\}$. These figures show that $\langle C\rangle$ increases with $K$, whereas $\mathcal{P}_{\text {out }}$ decreases inversely with $K$. This observation reveals that the performance of the system can be improved as the number of users increases. In addition, if there is no direct link S-E (i.e., $\Omega_{S E}=0$ ), then $\langle C\rangle$ reaches its peak at $P_{S}=P_{\max }$ and $\mathcal{P}_{\text {out }}$ gets minimum at $P_{S}=P_{\max }$ as well. However, this observation does not hold for the case of having direct link S-E (i.e., $\Omega_{S E} \neq 0$ ).

\section{CONCLUSIONS}

In this work, we have considered a secured cooperative relaying network with the best user selection technique. We have proposed the optimal transmit power allocation strategy to help the instantaneous SR attain its maximum. Besides, we have investigated the secure performance of the system through the average SR and the SOP.

\section{REFERENCES}

[1] Y. Zou, X. Wang, and W. Shen, "Optimal relay selection for physicallayer security in cooperative wireless networks," IEEE J. Sel. Areas Commun., vol. 31, no. 10, pp. 2099-2111, Oct. 2013.

[2] J. Yang, I.-M. Kim, and D. I. Kim, "Joint design of optimal cooperative jamming and power allocation for linear precoding," IEEE Trans. Commun., vol. 62, no. 9, pp. 3285-3298, Sep. 2014.

[3] _ - "Optimal cooperative jamming for multiuser broadcast channel with multiple eavesdroppers," IEEE Trans. Wireless Commun., vol. 12, no. 6, pp. 2840-2852, Jun. 2013.

[4] H. Alves, R. D. Souza, M. Debbah, and M. Bennis, "Performance of transmit antenna selection physical layer security schemes," IEEE Signal Process. Lett., vol. 19, no. 6, pp. 372-375, Jun. 2012.

[5] L. Fan, X. Lei, T. Q. Duong, M. Elkashlan, and G. K. Karagiannidis, "Secure multiuser communications in multiple amplify-and-forward relay networks," IEEE Trans. Commun., vol. 62, no. 9, pp. 3299-3310, Sep. 2014.

[6] V. N. Q. Bao, N. L. Trung, and M. Debbah, "Relay selection schemes for dual-hop networks under security constraints with multiple eavesdroppers," IEEE Trans. Wireless Commun., vol. 12, no. 12, pp. 6076-6085, Dec. 2013.

[7] R. U. Nabar, H. Bolcskei, and F. W. Kneubuhler, "Fading relay channels: Performance limits and space-time signal design," IEEE J. Sel. Areas Commun., vol. 22, no. 6, pp. 1099-1109, Aug. 2004.

[8] T. M. Cover and J. A. Thomas, Elements of Information Theory, 2nd ed. USA: Wiley, 2006.

[9] B. Suard, G. Xu, H. Liu, and T. Kailath, "Uplink channel capacity of space-division-multiple-access schemes," IEEE Trans. Inf. Theory, vol. 44, no. 4, pp. 1468-1476, Jul. 1998.

[10] R. D. Yates and D. J. Goodman, Probability and stochastic processes: A friendly introduction for electrical and computer engineers, 2 nd ed. USA: Wiley, 2004.

[11] I. S. Gradshteyn and I. M. Ryzhik, Table of Integrals, Series, and Products. USA: Academic Press, 2007.

$$
\begin{array}{r}
\langle C\rangle=(2 \ln 2)^{-1}\left(\bar{\gamma}_{S E}-\bar{\gamma}_{R E}\right)^{-1} e^{\frac{1}{\bar{\gamma}_{S R}}} \widetilde{\sum_{k}} e^{\frac{k}{\bar{\gamma}_{R D}}}\left\{\bar{\gamma}_{R E}\left[e^{\frac{1}{\bar{\gamma}_{R E}}} E_{1}\left(k / \bar{\gamma}_{R D}+1 / \bar{\gamma}_{S R}+1 / \bar{\gamma}_{R E}\right)-E_{1}\left(k / \bar{\gamma}_{R D}+1 / \bar{\gamma}_{S R}\right)\right]\right. \\
\left.-\bar{\gamma}_{S E}\left[e^{\frac{1}{\bar{\gamma}_{S E}}} E_{1}\left(k / \bar{\gamma}_{R D}+1 / \bar{\gamma}_{S R}+1 / \bar{\gamma}_{S E}\right)-E_{1}\left(k / \bar{\gamma}_{R D}+1 / \bar{\gamma}_{S R}\right)\right]\right\} .
\end{array}
$$

